\title{
Optimization of cooperative spectrum sensing with sensing user selection in cognitive radio networks
}

\author{
Huogen $\mathrm{Yu}^{*}$, Wanbin Tang and Shaoqian Li
}

\begin{abstract}
Cooperative spectrum sensing (CSS) can improve the spectrum sensing performance by introducing spatial diversity in cognitive radio networks (CRNs). However, such cooperation also introduces the delay for reporting sensing data. Conventional cooperation scheme assumes that the cooperative secondary users (SUs) report their local sensing data to the fusion center sequentially. This causes the reporting delay to increase with the number of the cooperative SUs, and ultimately affects the performance of CSS. In this article, we consider the reporting delay and formulate the optimization problem of CSS with sensing user selection to maximize the average throughput of the CRN in both the additive white Gaussian noise (AWGN) environment and the Rayleigh fading environment. It is shown that selecting all the SUs within the CRN to cooperate might not achieve the maximal average throughput. In particular, for the AWGN environment, the sensing user selection scheme is equivalent to selecting the optimal number of cooperative SUs due to all the SUs having the same instantaneous detection signal-to-noise ratio (SNR). For the Rayleigh fading environment, the maximal average throughput is achieved by selecting a certain number of cooperative SUs with the highest instantaneous detection SNRs to cooperate. Finally, computer simulations are presented to demonstrate that the average throughput of the CRN can be maximized through the optimization.
\end{abstract}

Keywords: cooperative spectrum sensing, cognitive radio, reporting delay, optimization, sensing user selection

\section{Introduction}

Cognitive radio (CR) technology has recently been identified as a promising way to address the spectrum scarcity by exploiting opportunistic spectrum in dynamically changing environments [1,2]. A prerequisite of CR is the ability to detect very weak primary user (PU) signals and limit the probability of interference with PU. Thus, spectrum sensing plays an essential role in CR. However, due to multipath fading, the shadow effect and time-varying natures of wireless channels, it is hard to achieve reliable spectrum sensing by a single secondary user (SU). To combat these impacts, cooperative spectrum sensing (CSS) has been proposed to improve the spectrum sensing performance by introducing spatial diversity [3-14]. There are mainly two fusion rules of

\footnotetext{
* Correspondence: yuhuogen@uestc.edu.cn University of Electronic Science and Technology of China, National Key Laboratory of Science and Technology on Communications, Chengdu 611731, China
}

\section{Springer}

(c) 2011 Yu et al; licensee Springer. This is an Open Access article distributed under the terms of the Creative Commons Attribution License (http://creativecommons.org/licenses/by/2.0), which permits unrestricted use, distribution, and reproduction in any medium, provided the original work is properly cited.
CSS: data fusion rule and decision fusion rule. In this article, we focus on the data fusion rule. For the data fusion rule, multiple cooperative SUs individually sense the channel, and then report their local sensing data to the fusion center through a bandwidth-limited common control channel. Finally, the fusion center will combine these data and make the final decision.

The sensing time, the data fusion rule and the fusion rule's threshold at the fusion center can all affect the performance of CSS. A longer sensing time will improve the spectrum sensing performance, but decrease the data transmission time. Moreover, an optimal data fusion rule can help reduce the impact of unreliable CR. In [8-10], the optimal linear functions of weighed data fusion rule in different cases have been obtained. In [12], a joint optimization of the sensing time and data fusion rule is considered.

However, in order to apply CSS, local sensing data have to be reported to the fusion center through a 
bandwidth-limited common control channel. This adds the reporting delay to cognitive radio networks (CRNs). To address this issue, the study [15] proposed that cooperative SUs sent local sensing data concurrently. But this scheme will increase the system design complexity or cost a large portion of precious bandwidth. Therefore, given a bandwidth-limited common control channel, the conventional scheme that cooperative SUs report their local sensing data to the fusion center sequentially may be more desirable [16]. Nevertheless, in the conventional scheme, the reporting delay increases with the number of cooperative SUs, which will lead to the decrease of the time for spectrum sensing and data transmission. Thus, there is a tradeoff between the number of cooperative SUs and the average throughput of the CRN. In [17], the authors demonstrated that selecting all SUs to cooperate in the CRN might not achieve the optimum performance. So they proposed a sensing user selection scheme based on the individual characteristics. But the sensing time was not considered in their optimization formulation.

In this article, we consider the conventional scheme that cooperative SUs report the local sensing data to the fusion center sequentially. We formulate the optimization problem of CSS with sensing user selection in both the additive white Gaussian noise (AWGN) environment and the Rayleigh fading environment. It is demonstrated that the maximal average throughput is achieved through the optimization. It is also shown that the maximal average throughput might be achieved by selecting a certain number of cooperative SUs rather than selecting all the SUs within the CRN.

The rest of the article is organized as follows: The system model is introduced in Section 2. The problem formulation based on data fusion rule is given in Section 3, and in Section 4, the solution of the optimization problem is presented. Numerical results and discussions are given in Section 5. Finally, conclusions are drawn in Section 6.

\section{System model}

Without loss of generality, we consider a CRN with $N$ SUs among which $k(1 \leq k \leq N, k \in I, I$ is the set of all positive intergers) SUs are employed to cooperate to sense a PU channel. There is a fusion center in the CRN, which assigns $k$ SUs to cooperate to sense the PU channel through the sensing user selection scheme and collects spectrum sensing information from the $k$ SUs through a common control channel. Similar to $[13,18,19]$, we assume that the size of the CRN is small compared with its distance from the primary system. Therefore, the received signal at each SU experiences almost identical path loss. Note, however, the results obtained in this article can be easily generalized to the case that the received signal at each SU experiences different path loss.

A frame structure is designed with periodic spectrum sensing for the secondary system. Figure 1 shows the frame structure considered for the periodic spectrum sensing. There are three phases in each frame: a sensing phase, a reporting phase, and a data transmission phase. In the sensing phase, all the cooperative SUs perform local spectrum sensing simultaneously. In the reporting phase, the local sensing data are reported to the fusion center sequentially. In the data transmission phase, data of SUs are transmitted. We assume that the durations of the sensing phase and the reporting delay of each cooperative SUs are respectively denoted as $\tau_{s}$ and $\tau_{r}$.

For ease of presentation in this article, we further assume that the primary system and the secondary system use a synchronous frame structure. During each frame of duration $T$, the PU on the channel is either

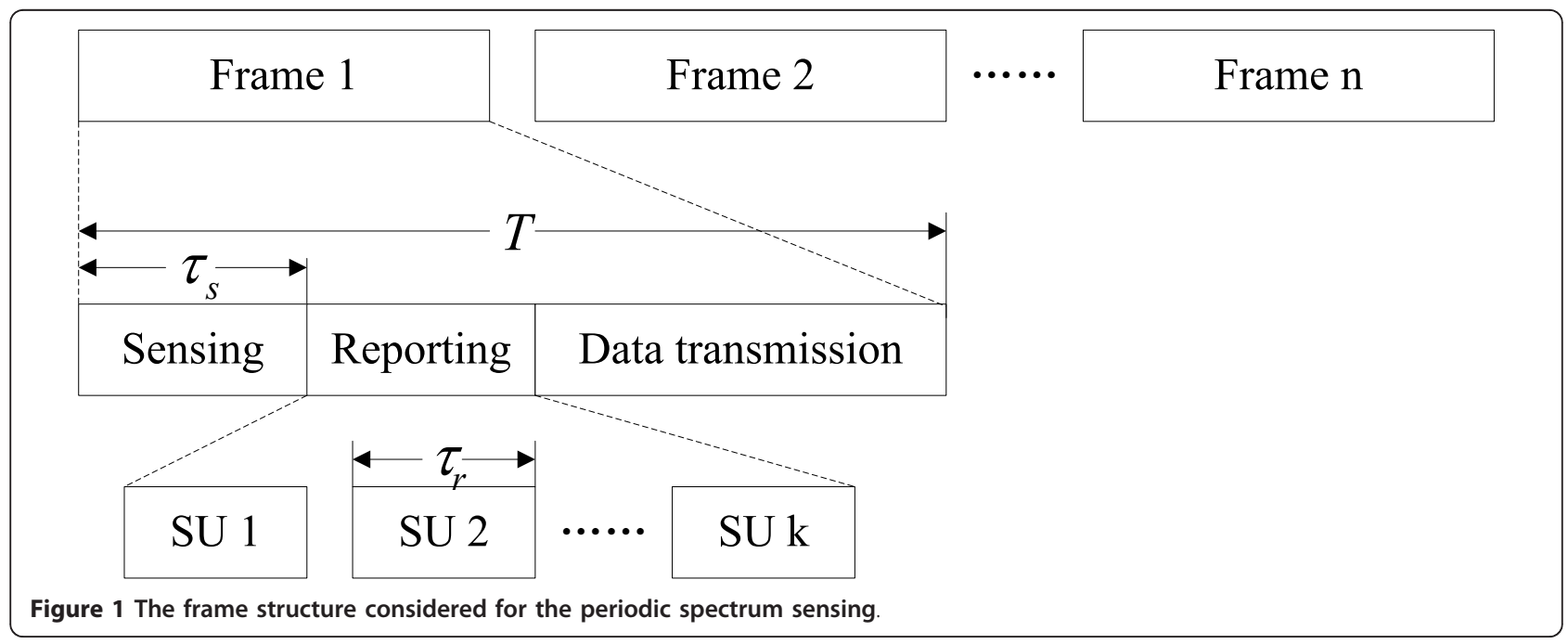


absent or present. The assumption has been widely used in e.g., $[12,20-22]$. It is easy to see that the performance of spectrum sensing will significantly degraded for the asynchronous frame structure, and the CRN's maximum average throughput we obtain in this article will provide an upper bound.

The most important motivation of $\mathrm{CR}$ is to improve the spectrum efficiency. Therefore, reporting overhead in CR system cannot be large, which means using a wideband common control channel to transmit the local sensing data is not feasible. Due to the constraint of common control channel bandwidth, the local sensing data should be quantized before reporting to the fusion center. We assume that the bandwidth of common control channel is given as $\dot{B}$, and the quantizer can well preserve the local sensing data with $q$ quantization bits. When the binary phase shift keying modulation is adopted, the reporting delay of each cooperative $\mathrm{SU}$ is [17]

$$
\tau_{r}=\frac{q}{B} .
$$

The increase of cooperative SU's number leads to a high space diversity gain and helps to improve the spectrum sensing performance. However, it also results in the increase of total reporting delay which leads to the decrease of the spectrum sensing time and data transmission time. Hence, there exists a tradeoff between the number of cooperative SUs and the average throughput of the CRN.

\subsection{Energy detection}

Local spectrum sensing problem can be formulated as a binary hypothesis test between the following two hypotheses:

$$
\begin{aligned}
& H_{0}: y_{i}(n)=u_{i}(n), \quad n=1,2, \ldots, \tau_{s} f_{s} \\
& H_{1}: y_{i}(n)=h_{i} s_{i}(n)+u_{i}(n), \quad n=1,2, \ldots, \tau_{s} f_{s}
\end{aligned}
$$

where $H_{0}$ and $H_{1}$ denote that the PU on the channel is absent and present respectively. $y_{i}(n)$ represents the received signal at the $i$ th $\mathrm{SU} . h_{i}$ denotes the channel coefficient from the PU to the $i$ th $\mathrm{SU}$, which is assumed to be constant during the sensing phase [13]. $s_{i}(n)$ is the signal transmitted from the PU. The noise $u_{i}(n)$ is the circular symmetric complex Gaussian signal with mean zero and variance $\sigma_{u}^{2} \cdot f_{s}$ is the sampling frequency. We assume that $s_{i}(n)$ is a complex-valued phase-shift keying signal with $\sigma_{s}^{2}$ denoting the signal power. The instantaneous detection signal-to-noise ratio (SNR) at the $i$ th $\mathrm{SU}$ is given as $\gamma_{i}=\frac{\left|h_{i}\right|^{2} \sigma_{s}^{2}}{\sigma_{u}^{2}}$. Herein, we also assume that the fusion center has perfect knowledge of the instantaneous detection SNR $\gamma_{i}$, and this can be realized by direct feedback from the SUs.

The AWGN environment and the Rayleigh fading environment are considered in this article. For the AWGN environment, all the SUs have the same channel coefficient $h_{i}$ due to all the SUs having identical path loss. Therefore, the instantaneous detection SNRs of all SUs are the same $\left(\gamma_{1}=\gamma_{2}=\mathrm{L}=\gamma_{i}=\gamma\right)$ in the AWGN environment. For the Rayleigh fading environment, the channel coefficients $\left|h_{i}\right|^{2}$ follow the exponential distribution, and have the same mean due to all the SUs having identical path loss. Therefore, the instantaneous detection SNRs of all SUs are exponentially distributed random variables with the same mean $\bar{\gamma}$ in the Rayleigh fading environment.

In this article, we concentrate on energy detection due to its ability to detect PU without prior information. Based on the energy detection, the test statistic of the $i$ th SU's received signal energy on the channel can be expressed as

$$
V_{i}=\frac{1}{\tau_{s} f_{s}} \sum_{n=1}^{\tau_{s} f_{s}}\left|\gamma_{i}(n)\right|^{2}
$$

For a large $\tau_{s} f_{s}, V_{i}$ can be approximated ${ }^{1}$ as the following Gaussian distribution according to the central limit theorem [12],

$$
V_{i} \sim \begin{cases}N\left(\sigma_{u}^{2}, \frac{1}{\tau_{s} f_{s}} \sigma_{u}^{4}\right), & H_{0} \\ N\left(\sigma_{u}^{2}\left(1+\gamma_{i}\right), \frac{1}{\tau_{s} f_{s}} \sigma_{u}^{4}\left(1+2 \gamma_{i}\right)\right) . & H_{1}\end{cases}
$$

\subsection{Data fusion rule}

In the data fusion rule, the test statistic of cooperative SU's received signal energy will be reported to the fusion center and will be summed with weighs by the fusion center. Finally, the fusion center will make the final decision based on the weighed summation.

Denote the weigh coefficient corresponding to the $i$ th cooperative $\mathrm{SU}$ to be $w_{i}$, then the test statistic used for final decision is given by

$$
V=\sum_{i=1}^{k} w_{i} V_{i}
$$

where $k$ is the number of SUs assigned to cooperate to sense the PU channel. Without loss of generality, we assume that $\sum_{i=1}^{k} w_{i}^{2}=1$. Similar to the study [12], we can prove that $V$ is Gaussian with

$$
V \sim \begin{cases}N\left(\sigma_{u}^{2} \sum_{i=1}^{k} w_{i}, \frac{1}{\tau_{s}} \sigma_{s}^{4}\right), & H_{0} \\ N\left(\sigma_{u}^{2} \sum_{i=1}^{k} w_{i}\left(1+\gamma_{i}\right), \frac{1}{\tau_{s} f_{s}} \sigma_{u}^{4} \sum_{i=1}^{k} w_{i}^{2}\left(1+2 \gamma_{i}\right)\right) . & H_{1}\end{cases}
$$


If we choose the decision threshold as $\varepsilon$, the probabilities of false alarm and detection are given by

$$
\begin{aligned}
& P_{f}\left(k,\left\{w_{i}\right\}, \tau_{s}, \varepsilon\right)=Q\left(\frac{\varepsilon-\sigma_{u}^{2} \sum_{i=1}^{k} w_{i}}{\sigma_{u}^{2}} \sqrt{\tau_{s} f_{s}}\right), \\
& P_{d}\left(k,\left\{w_{i}\right\},\left\{\gamma_{i}\right\}, \tau_{s}, \varepsilon\right)=Q\left(\frac{\varepsilon-\sigma_{u}^{2} \sum_{i=1}^{k} w_{i}\left(1+\gamma_{i}\right)}{\sigma_{u}^{2} \sqrt{\sum_{i-1}^{k} w_{i}^{2}\left(1+2 \gamma_{i}\right)}} \sqrt{\tau_{s} f_{s}}\right),
\end{aligned}
$$

respectively, where $Q(\cdot)$ is the complementary distribution function of the standard Gaussian. The parameter selection of $\left\{k,\left\{w_{i}\right\},\left\{\gamma_{i}\right\}\right\}$ depends on the sensing user selection scheme.

Proposition 1: Suppose the low instantaneous detection SNR regime is of interest. For a target detection probability $P_{d}$, the optimal values of $\left\{w_{i}\right\}$ with specific $k$, $\left\{\gamma_{i}\right\}$, and $\tau_{s}$ are given by

$$
w_{i}^{*}=\frac{\gamma_{i}}{\sqrt{\sum_{i-1-1}^{k} \gamma_{i}^{2}}} . \quad 1 \leq i \leq k
$$

Proof: The proof is similar to that in [[12], Theorem $2]$. In here, we only provide a brief proof.

By combining (8) and (9), $P_{f}$ can be expressed as

$$
P_{f}\left(k,\left\{w_{i}\right\},\left\{\gamma_{i}\right\}, \tau_{s}\right)=Q\left(Q^{-1}\left(P_{d}\right) \sqrt{\sum_{i=1}^{k} w_{i}^{2}\left(1+2 \gamma_{i}\right)}+\sqrt{\tau_{s} f_{s}} \sum_{i=1}^{k} w_{i} \gamma_{i}\right) .
$$

In the context of CR, the PU's signal power received by the SUs is usually very low [24]. Thus, we are interested in the low instantaneous detection SNR regime where $\gamma_{i} \ll 1$. In this case, $\sqrt{\sum_{i=1}^{k} w_{i}^{2}\left(1+2 \gamma_{i}\right)} \approx 1$ and $P_{f}$ can be approximated as

$$
P_{f}\left(k,\left\{w_{i}\right\},\left\{\gamma_{i}\right\}, \tau_{s}\right) \approx Q\left(Q^{-1}\left(P_{d}\right)+\sqrt{\tau_{s} f_{s}} \sum_{i=1}^{k} w_{i} \gamma_{i}\right) .
$$

Therefore, for specific $k,\left\{\gamma_{i}\right\}$, and $\tau s$, the optimal $\left\{w_{i}\right\}$ is designed to achieve minimum probability of $P_{f}$ :

$$
\underset{\left\{w_{i}\right\}, \sum_{i=1}^{k} w_{i}^{2}=1}{\arg \min } P_{f} .
$$

Obviously, (13) is equivalent to the following optimization function:

$$
\underset{\left\{w_{i}\right\}, \sum_{i=1}^{k} w_{i}^{2}=1}{\arg \max } \sum_{i=1}^{k} w_{i} \gamma_{i} .
$$

Using Cauchy-Schwarz inequality, we obtain the optimal values of $\left\{w_{i}\right\}$ with specific $k,\left\{\gamma_{i}\right\}$, and $\tau_{\mathrm{s}}$ given by (10).

\section{Problem formulation}

In this section, we consider the reporting delay and formulate the optimization problem of CSS with sensing user selection to maximize the average throughput of the CRN in both the AWGN environment and the Rayleigh fading environment.

There are two scenarios for which the CRN can operate on the channel [12]: 1) the PU is absent and no false alarm is generated by the fusion center, 2) the PU is present but it is not detected by the fusion center. We denote $C_{0}$ and $C_{1}$ as the throughput of the CRN if they are allowed to operate in the absence and presence of the PU, respectively. Then the average throughput of the $\mathrm{CRN}$ for the two scenarios can be given respectively as

$$
\begin{aligned}
& R_{0}\left(k,\left\{w_{i}\right\}, \tau_{s}, \varepsilon\right)=\frac{T-\tau_{s}-k \tau_{r}}{T} P\left(H_{0}\right)\left[1-P_{f}\left(k,\left\{w_{i}\right\}, \tau_{s}, \varepsilon\right)\right] C_{0}, \\
& R_{1}\left(k,\left\{w_{i}\right\},\left\{\gamma_{i}\right\}, \tau_{s}, \varepsilon\right)=\frac{T-\tau_{s}-k \tau_{r}}{T} P\left(H_{1}\right)\left[1-P_{d}\left(k,\left\{w_{i}\right\},\left\{\gamma_{i}\right\}, \tau_{s}, \varepsilon\right)\right] C_{1},
\end{aligned}
$$

where $P\left(H_{0}\right)$ and $P\left(H_{1}\right)$ are probabilities that the PU is absent and present, respectively.

In order to maximize the average throughput of the CRN, the optimization problem is formulated as follows: Problem P1:

$$
\begin{aligned}
& \max _{k,\left\{w_{i}\right\},\left\{\gamma_{i}\right\}, \tau_{s}, \varepsilon} R\left(k,\left\{w_{i}\right\},\left\{\gamma_{i}\right\}, \tau_{s}, \varepsilon\right)=R_{0}\left(k,\left\{w_{i}\right\}, \tau_{s}, \varepsilon\right)+R_{1}\left(k,\left\{w_{i}\right\},\left\{\gamma_{i}\right\}, \tau_{s}, \varepsilon\right) \\
& \text { s.t. } \quad 1 \leq k \leq N, \quad k \in I \\
& P_{d}\left(k,\left\{w_{i}\right\},\left\{\gamma_{i}\right\}, \tau_{s}, \varepsilon\right) \geq \text { Pth } \\
& 0 \leq \tau_{s}+k \tau_{r} \leq T \\
& \sum_{i=1}^{k} w_{i}^{2}=1
\end{aligned}
$$

It can be proved that the optimal solution of problem P1 occurs when $P_{d}\left(k,\left\{w_{i}\right\},\left\{\gamma_{i}\right\}, \tau_{s}, \varepsilon\right)=$ Pth. The proof is similar to that in [25]. In here, we only provide a brief explanation. For specific $k,\left\{w_{i}\right\},\left\{\gamma_{i}\right\}$, and $\tau_{s}$, the values of $P_{d}\left(k,\left\{w_{i}\right\},\left\{\gamma_{i}\right\}, \tau_{s}, \varepsilon\right)$ and $P_{f}\left(k,\left\{w_{i}\right\}, \tau_{s}, \varepsilon\right)$ are inversely proportional to the sensing threshold $\varepsilon$. When $P_{d}\left(k,\left\{w_{i}\right\},\left\{\gamma_{i}\right\}, \tau_{s}, \varepsilon\right)$ is minimized, the sensing threshold $\varepsilon$ is maximized. From (17), it can be seen that the objective function is maximized when the sensing threshold $\varepsilon$ is maximized. Hence, the sensing threshold $\varepsilon$ should always be chosen to meet the minimum requirement of $P_{d}\left(k,\left\{w_{i}\right\},\left\{\gamma_{i}\right\}, \tau_{s}, \varepsilon\right)=$ Pth.

Meanwhile, for a target detection probability $P_{d}(k$, $\left.\left\{w_{i}\right\},\left\{\gamma_{i}\right\}, \tau_{s}, \varepsilon\right)=P$ th , we can know that problem P1 achieves the optimal solution when according to the Proposition 1.

\subsection{AWGN environment}

In the AWGN environment, all the SUs have the same instantaneous detection SNR. So we have 


$$
w_{i}=w_{i}^{*}=\frac{1}{\sqrt{k}}, 1 \leq i \leq k
$$

Therefore, $\varepsilon$ and $P_{f}$ of problem P1 can be expressed as

$$
\begin{aligned}
& \varepsilon\left(k, \tau_{s}\right)=\sigma_{u}^{2}\left(Q^{-1}(P t h) \sqrt{\frac{1+2 \gamma}{\tau_{s} f_{s}}}+\sqrt{k}+\gamma \sqrt{k}\right), \\
& P_{f}\left(k, \tau_{s}\right)=Q\left(Q^{-1}(P t h) \sqrt{1+2 \gamma}+\gamma \sqrt{k \tau_{s} f_{s}}\right) .
\end{aligned}
$$

For the AWGN environment, sensing user selection is equivalent to selecting the optimal number of cooperative SUs due to all the SUs having the same instantaneous detection SNR. Then, the problem $\mathrm{Pl}$ is equivalent to the following problem in the AWGN environment:

Problem P2:

$$
\begin{aligned}
& \max _{k, \tau_{s}} \quad R\left(k, \tau_{s}\right)=\frac{T-\tau_{s}-k \tau_{\tau}}{T}\left\{P\left(H_{0}\right)\left[1-P_{f}\left(k, \tau_{s}\right)\right] C_{0}+P\left(H_{1}\right)[1-P t h] C_{1}\right\} \\
& \text { s.t. } \quad 1 \leq k \leq N, \quad k \in I \\
& 0 \leq \tau_{s}+k \tau_{r} \leq T
\end{aligned}
$$

\subsection{Rayleigh fading environment}

In the Rayleigh fading environment, $\varepsilon$ and $P_{f}$ of problem P1 can be expressed as

$$
\begin{gathered}
\varepsilon\left(k,\left\{\gamma_{i}\right\}, \tau_{s}\right)=\sigma_{u}^{2}\left(Q^{-1}(P t h) \sqrt{\frac{1+2 \frac{\sum_{i=1}^{k} \gamma_{i}^{3}}{\sum_{i=1}^{i} \gamma_{s}^{2}}}{\tau_{s}}}+\frac{\sum_{i=1}^{k} \gamma_{i}}{\sqrt{\sum_{i=1}^{k} \gamma_{i}^{2}}}+\sqrt{\sum_{i=1}^{k} \gamma_{i}^{2}}\right) \\
P_{f}\left(k,\left\{\gamma_{i}\right\}, \tau_{s}\right)=Q\left(Q^{-1}(P t h) \sqrt{1+2 \frac{\sum_{i=1}^{k} \gamma_{i}^{3}}{\sqrt{\sum_{i=1}^{k} \gamma_{i}^{2}}}}+\sqrt{\tau_{s} f_{s} \sum_{i=1}^{k} \gamma_{i}^{2}}\right) \\
\stackrel{\gamma_{i} \ll 1}{\approx} Q\left(Q^{-1}(P t h)+\sqrt{\tau_{s} f_{s} \sum_{i=1}^{k} \gamma_{i}^{2}}\right) .
\end{gathered}
$$

Therefore, the problem P1 is equivalent to the following problem in the Rayleigh fading environment:

Problem P3:

$$
\begin{aligned}
& \max _{k_{i}, \gamma_{i}, \tau_{s}} R\left(k,\left\{\gamma_{i}\right\}, \tau_{s}\right)=\frac{T-\tau_{s}-k \tau_{\tau}}{T}\left\{P\left(H_{0}\right)\left[1-P_{f}\left(k,\left\{\gamma_{i}\right\}, \tau_{s}\right)\right] C_{0}+P\left(H_{1}\right)[1-P t h] C_{1}\right\} \\
& \text { s.t. } \quad 1 \leq k \leq N, \quad k \in I \\
& 0 \leq \tau_{s}+k \tau_{r} \leq T
\end{aligned}
$$

Proposition 2: For given $k$ and $\tau_{s}$, the maximum average throughput $R\left(k,\left\{\gamma_{i}\right\}, \tau_{s}\right)$ can be achieved when $k$ SUs with the highest detection SNRs are selected to cooperate to sense the PU channel.
Proof: Let $\Omega=\left[\gamma_{1}, \gamma_{2}, \ldots, \gamma_{N}\right]$ denote the detection SNRs of the $N$ SUs. $\Omega^{\prime}=\left[\gamma_{n_{1}}, \gamma_{n_{2}}, \ldots, \gamma_{n_{N}}\right]\left(\gamma_{n_{1}} \geq \gamma_{n_{2}} \geq \cdots \geq \gamma_{n_{N}}\right)$ is a descending order of $\Omega$.

Firstly, when $k=1$, since $P_{f}\left(1,\left\{\gamma_{n_{1}}\right\}, \tau_{s}\right)$ can achieve the minimum value, $R\left(1,\left\{\gamma_{n_{1}}\right\}, \tau_{s}\right)$ can achieve maximum value.

Next, when $k=2$, we can note that

$$
\begin{aligned}
\gamma_{n_{1}} \geq \gamma_{n_{2}} \geq \cdots \geq \gamma_{n_{N}} & \Rightarrow \gamma_{n_{1}}^{2} \geq \gamma_{n_{2}}^{2} \geq \cdots \geq \gamma_{n_{N}}^{2} \\
& \Rightarrow \gamma_{n_{1}}^{2}+\gamma_{n_{2}}^{2}=\max \left(\gamma_{n_{i}}^{2}+\gamma_{n_{j}}^{2}\right) . \quad 1 \leq i, j \leq N, i \neq j
\end{aligned}
$$

Obviously, $Q^{-1}(P t h)+\sqrt{\tau_{s} f_{s}\left(\gamma_{n_{1}}^{2}+\gamma_{n_{2}}^{2}\right)}$ can achieve the maximum value when $k=2$. Using the fact that $Q(\cdot)$ is a decreasing function, it can be easily seen that $P_{f}\left(2,\left\{\gamma_{n_{1}}, \gamma_{n_{2}}\right\}, \tau_{s}\right)$ can achieve the minimum value. Therefore, $R\left(2,\left\{\gamma_{n_{1}}, \gamma_{n_{2}}\right\}, \tau_{s}\right)$ can achieve maximum value.

Then, in the same way, we can prove that the maximum average throughput $R\left(k,\left\{\gamma_{i}\right\}, \tau_{s}\right) \quad(3 \leq k \leq N, k \in I)$ can be achieved when $k$ SUs with the highest detection SNRs are selected to cooperate to sense the PU channel.

According to the Proposition 2, we can know that $\left\{\gamma_{i}\right\}$ is determined when $k$ is given.

\section{The solution of the optimization problem}

Instead of solving the problem P2 or P3 directly, we propose the algorithm that solves the problem P2 or P3 by an exhaustive search for $k$. Since $k$ is an integer and lies within the interval $[1, N]$, it is not computationally expensive to search.

In order to solve problem P2 or P3, we transform problem P2 or P3 to

$$
\begin{aligned}
& \max _{k} \quad R(k)=C^{*}(k) \\
& \text { s.t. } \quad 1 \leq k \leq N, \quad k \in I
\end{aligned}
$$

where $C^{*}(k)$ is the optimal objective value of the following problem $\mathrm{P} 4$ with a specific $k$ value.

Problem P4 (with a specific $k$ value):

$$
\begin{aligned}
& \max _{\tau_{s}} C\left(\tau_{s}\right)=\frac{T-\tau_{s}-k \tau_{r}}{T}\left\{P\left(H_{0}\right)\left[1-P_{f}\left(\tau_{s}\right)\right] C_{0}+P\left(H_{1}\right)[1-P t h] C_{1}\right\} \\
& \text { s.t. } \quad 0 \leq \tau_{s}+k \tau_{r} \leq T
\end{aligned}
$$

The optimization problem P4 is a convex optimization problem only if the following constraint should be satisfied [12]:

$$
P_{f}\left(\tau_{s}\right) \leq \frac{1}{2} .
$$

Obviously, the constraint in (38) is very reasonable for practical CR systems. 
Table 1 The solution of the optimization problem in the AWGN environment.

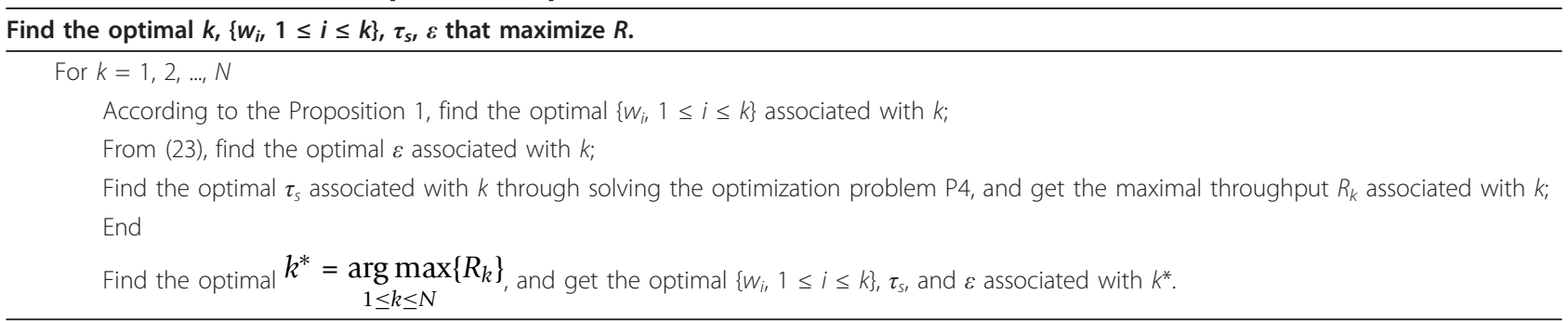

Finally, the solutions of the optimization problem in the AWGN environment and the Rayleigh fading environment are respectively presented in Tables 1 and 2 .

\section{Numerical results and discussions}

In this section, numerical results and discussions are presented to demonstrate the effectiveness of our proposed algorithms. The system is set up as follows: The number of SUs in the CRN is set to be $N=30$, and the fixed frame of $T=20 \mathrm{~ms}$ is used. The PU absent probability on the channel is $P\left(H_{0}\right)=0.7$. The sampling frequency is fixed at $6 \mathrm{MHz}$. The detection probability is $P$ th $=0.9$. Furthermore, we assume that the SU channel is block faded and $S N R_{S}$ (the SNR for secondary transmission) are ergodic, stationary, and exponentially distributed with the same mean $20 \mathrm{~dB}$. The SNR for PU measured at the secondary receiver is $S N R_{p}=\gamma$ in the AWGN environment or $S N R_{p}=\bar{\gamma}$ in the Rayleigh fading environment. Thus $C_{0}=$ $\log _{2}\left(1+S N R_{S}\right)$ and $C_{1}=\log _{2}\left(1+\frac{S N R_{S}}{1+S N R_{p}}\right)$. Since the $S N R_{S}$ can be different for different channel realizations, all the numerical results presented in this article are obtained by averaging over 10,000 independent simulation runs.

We first demonstrate several numerical results in the AWGN environment. Figure 2 shows the average throughput versus the number of cooperative SUs under different reporting delay when $\gamma=-20 \mathrm{~dB}$. It can be seen that the maximum average throughput might not be achieved when all the SUs within the CRN cooperate to sense the same PU channel. When the reporting delay is $\tau_{r}=0 \mathrm{~ms}$, the average throughput increases with increasing the number of cooperative SUs. But the growth of the average throughput is very slow when the number of cooperative SUs achieves a certain amount. When the reporting delay is $\tau_{r} \neq 0 \mathrm{~ms}$, the maximum average throughput first increases and then decreases as the number of cooperative SUs grows. Figure 3 shows the optimal number of cooperative SUs versus the reporting delay under different instantaneous detection SNR $\gamma$. It can be seen that the optimal number of cooperative SUs increases with decreasing the reporting delay and the instantaneous detection SNR. Figure 4 shows the optimal sensing time versus the reporting delay under different instantaneous detection SNR $\gamma$. It can be seen that the optimal sensing time increases with increasing the reporting delay and decreases with increasing the instantaneous detection SNR. Figure 5 shows the maximum average throughput versus the reporting delay under different instantaneous detection SNR $\gamma$. It is clear that the maximum average throughput decreases with increasing the reporting delay and increases with increasing the instantaneous detection SNR.

Next, we demonstrate numerical results in the Rayleigh fading environment. Figure 6 shows the average throughput versus the number of cooperative SUs under different reporting delay when the mean instantaneous detection SNR $\bar{\gamma}=-20 \mathrm{~dB}$. In Figure 6, when the number of cooperative SUs is equal to $k$, it says that $k$ SUs with the highest detection SNRs are selected to

Table 2 The solution of the optimization problem in the Rayleigh fading environment.

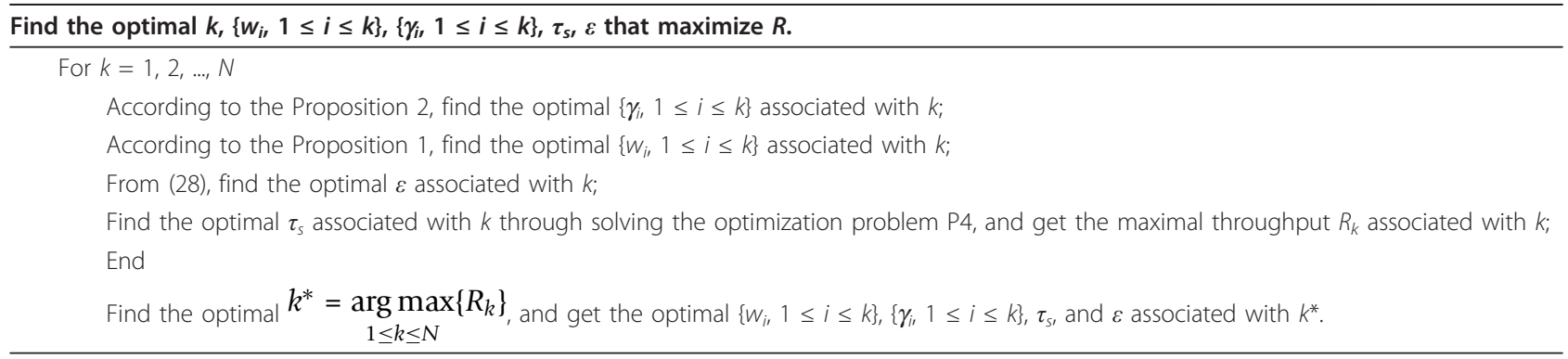




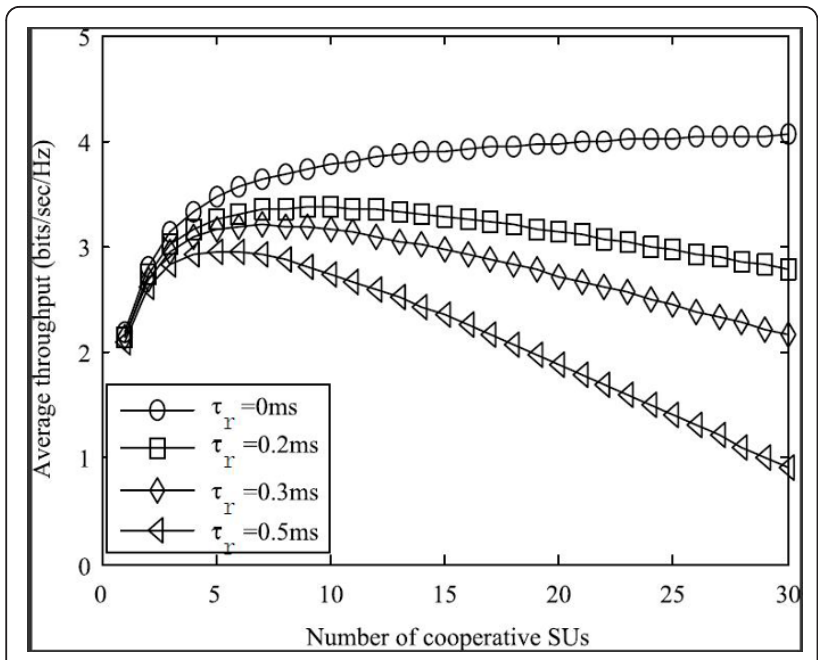

Figure 2 The average throughput versus the number of cooperative SUs in the AWGN environment.

cooperate to sense the PU channel. It can also be seen that the maximum average throughput might not be achieved when all the SUs within the CRN cooperate to sense the same PU channel. When the reporting delay is $\tau_{r}=0 \mathrm{~ms}$, the average throughput increases with increasing the number of cooperative SUs. But the growth of the average throughput is very slow when the number of cooperative SUs achieves a certain amount. When the reporting delay is $\tau_{r} \neq 0 \mathrm{~ms}$, the maximum average throughput first increases and then decrease as the number of cooperative SUs grows.

\section{Conclusion}

In this article, we have considered the influence of the reporting delay to the CSS and investigated the average

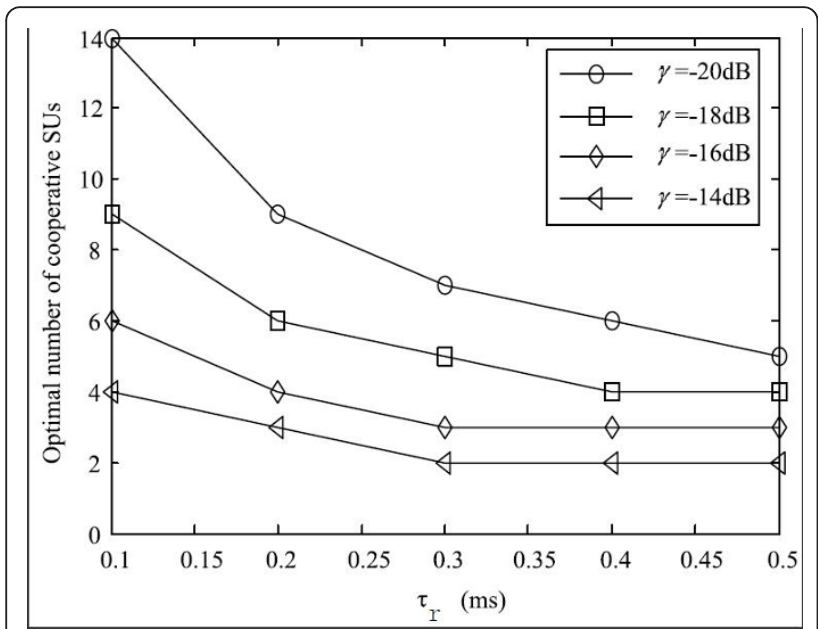

Figure 3 The optimal number of cooperative SUs versus the reporting delay in the AWGN environment.

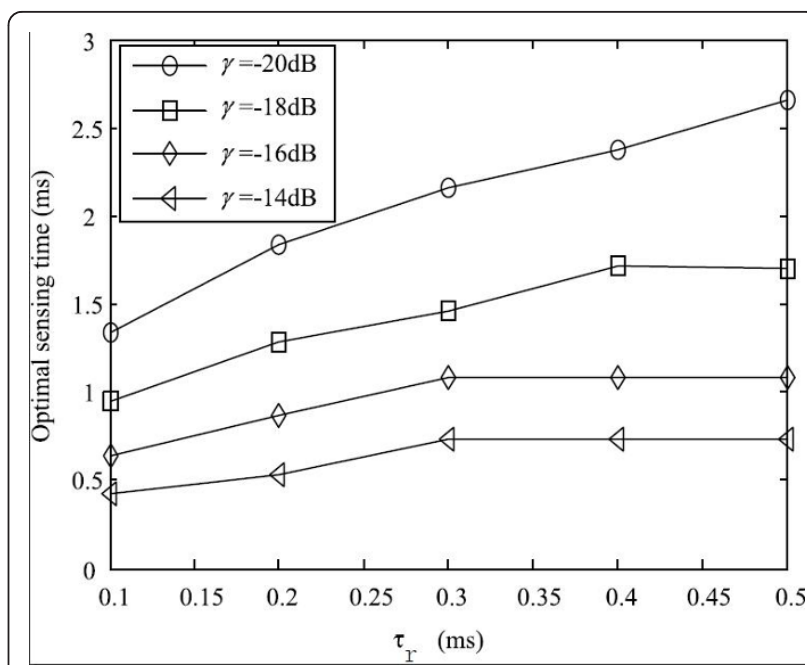

Figure 4 The optimal sensing time versus the reporting delay in the AWGN environment.

throughput problem under CSS scenario. The optimization problem of CSS with sensing user selection was formulated to maximize the average throughput of the CRN in both the AWGN environment and the Rayleigh fading environment, and the optimal solution was proposed to solve this problem. With numerical results, it is shown that the maximum average throughput can be achieved through the optimization. Moreover, it is also shown that selecting all the SUs within the CRN to cooperate might not obtain the maximal average throughput rather than selecting a certain number of SUs to cooperate.

\section{Endnote}

${ }^{1}$ To verify the accuracy of Gaussian approximation, the estimated probability density function (pdf) of energy

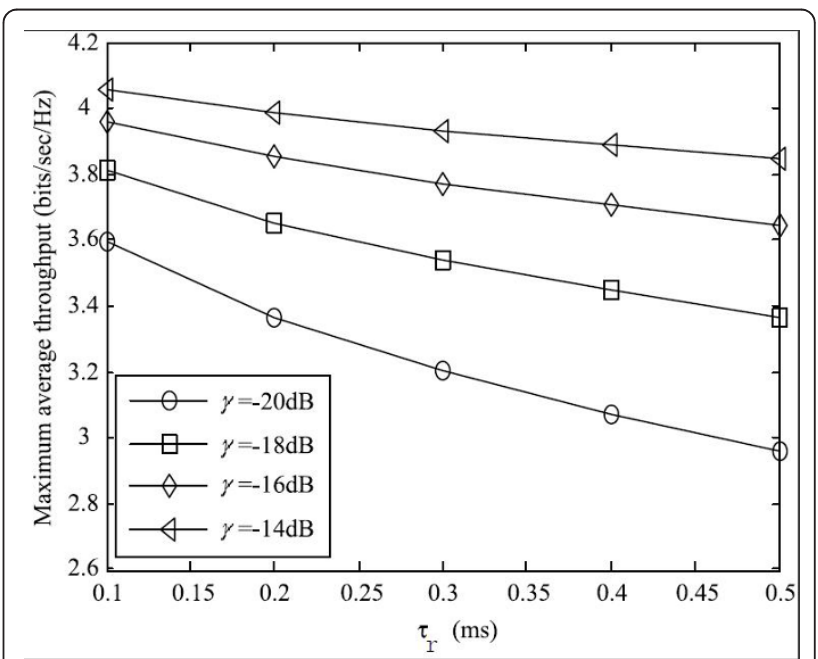

Figure 5 The maximum average throughput versus the reporting delay in the AWGN environment 


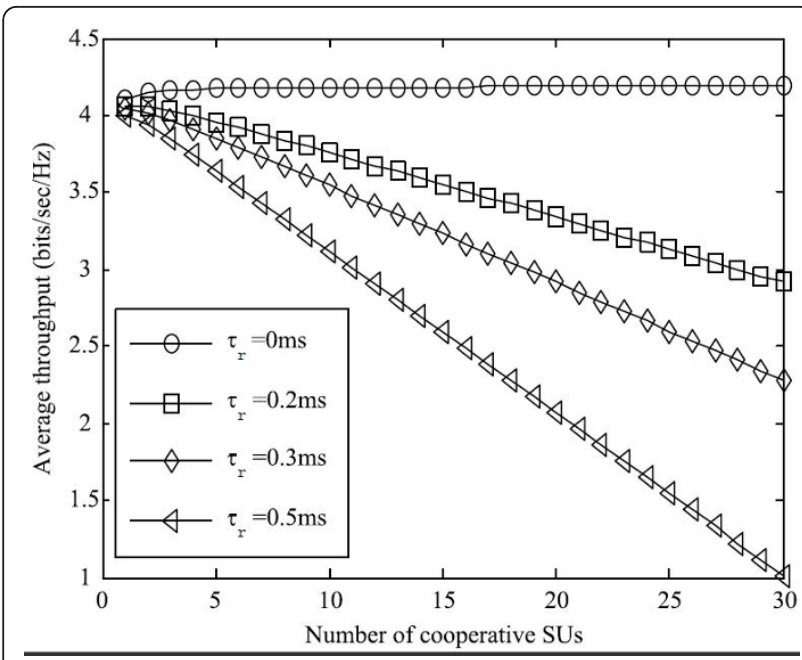

Figure 6 The average throughput versus the number of cooperative SUs in the Rayleigh fading environment.

measurements numerically obtained through MonteCarlo simulation was compared with the Gaussian pdf given by (5) [23]. The correlation between two pdfs was found to be greater than 0.99 for values of $\tau_{s} f_{s}$ as low as 50 for a wide range of $\gamma_{i}$ of practical interest.

\section{Acknowledgements}

This study was supported in part by National Basic Research Program (973 Program) of China under Grant No.2009CB320405, High-Tech Research and Development Program (863 Program) of China under Grant No.2009AA011801 and 2009AA012002, National Fundamental Research Program of China under Grant A1420080150, Nation Grand Special Science and Technology Project of China under Grant No.2008ZX03005001, 2009ZX03007-004, 2009ZX03005-002, 2009ZX03005-004, 2010ZX03006-002, 2009ZX03004001, 2010ZX03002-008-03 and National Natural Science Foundation of China under Grant No.61071102. The authors would like to thank the anonymous reviewers for their insightful comments and suggestions.

\section{Competing interests}

The authors declare that they have no competing interests.

Received: 27 April 2011 Accepted: 30 December 2011 Published: 30 December 2011

\section{References}

1. J Mitola, GQ Maguire, Cognitive radio: making software radios more personal. IEEE personal Commun J. 6(4), 13-18 (1999). doi:10.1109/ 98.788210

2. S Haykin, Cognitive radio: brain-empowered wireless communications. IEEE J Sel Areas Commun. 23(2), 201-220 (2005)

3. A Ghasemim, ES Sousa, Collaborative spectrum sensing for opportunistic access in fading environments. in Proceedings of the IEEE International
Symposium on New Frontiers in Dynamic Spectrum Access Networks (DySPAN 05), 131-136 (2005)

4. E Peh, Y-C Liang, Optimization for cooperative sensing in cognitive radio networks. in Proceedings of the IEEE Wireless Communications and Networking Conference (WCNC '07), 27-32 (2007)

5. G Ganesan, Y(G) Li, Cooperative spectrum sensing in cognitive radio, part I: two user networks. IEEE Trans Wirel Commun. 6(6), 2204-2213 (2007)

6. G Ganesan, $Y(G) L i$, Cooperative spectrum sensing in cognitive radio, part II: multiuser networks. IEEE Trans Wirel Commun. 6(6), 2214-2222 (2007)

7. J Ma, Y(G) Li, Soft combination and detection for cooperative spectrum sensing in cognitive radio networks. in Proceedings of the IEEE Global Telecommunications Conference (GLOBECOM '07), 3139-3143 (2007)

8. Z Quan, S Cui, AH Sayed, Optimal linear cooperation for spectrum sensing in cognitive radio networks. IEEE J Sel Top Signal Process. 2(1), 28-40 (2008)

9. Z Quan, W-K Ma, S Cui, AH Sayed, Optimal linear fusion for distributed detection via semidefinite programming. IEEE Trans Signal Process. 58(4), 2431-2436 (2010)

10. G Taricco, Optimization of linear cooperative spectrum sensing for cognitive radio networks. IEEE J Sel Top Signal Process. 5(1), 77-86 (2011)

11. KB Letaief, W Zhang, Cooperative communications for cognitive radio networks. Proc IEEE. 97(5), 878-893 (2009)

12. Y-C Liang, Y Zeng, ECY Peh, AT Hoang, Sensing-throughput tradeoff for cognitive radio networks. IEEE Trans Wirel Commun. 7(4), 1326-1337 (2008)

13. W Zhang, RK Mallik, KB Letaief, Optimization of cooperative spectrum sensing with energy detection in cognitive radio networks. IEEE Trans Wirel Commun. 8(12), 5761-5766 (2009)

14. X Zhou, J Ma, Y(G) Li, YH Kwon, ACK Soong, Probability-based combination for cooperative spectrum sensing. IEEE Trans Wirel Commun. 58(2), 463-466 (2010)

15. S Zhang, T Wu, VKN Lau, A low-overhead energy detection based cooperative sensing protocol for cognitive radio systems. IEEE Trans Wirel Commun. 8(11), 5575-5581 (2009)

16. F Gao, W Yuan, W Liu, W Cheng, S Wang, Pipelined cooperative spectrum sensing in cognitive radio networks. in Proceedings of the IEEE Wireless Communications and Networking Conference (WCNC '09), 1-5 (2009)

17. W Xia, W Yuan, W Cheng, W Liu, S Wang, J Xu, Optimization of cooperative spectrum sensing in ad-hoc cognitive radio networks. in Proceedings of the IEEE Global Telecommunications Conference (GLOBE- COM '10), 1-5 (2010)

18. Y Chen, Optimum number of secondary users in collaborative spectrum sensing considering resources usage efficiency. IEEE Commun Lett. 12(12), 877-879 (2008)

19. J Shen, T Jiang, S Liu, Z Zhang, Maximum channel throughput via cooperative spectrum sensing in cognitive radio networks. IEEE Trans Wirel Commun. 8(10), 5166-5175 (2009)

20. Q Zhao, L Tong, A Swami, Y Chen, Decentralized cognitive MAC for opportunistic spectrum access in ad hoc networks: a POMDP framework. IEEE J Sel Areas Commun. 25(3), 589-600 (2007)

21. Q Zhao, B Sadler, A survey of dynamic spectrum access. IEEE Signal Process Mag. 24(3), 79-89 (2007)

22. Q Zhao, S Geirhofer, L Tong, B Sadler, Opportunistic spectrum access via periodic channel sensing. IEEE Trans Signal Process. 36(2), 785-796 (2008)

23. AS Fotheringham, DC Knudsen, Goodness-of-Fit Statistics (Geo Books, Norwich, U.K, 1987)

24. D Cabric, SM Mishra, RW Brodersen, Implementation issues in spectrum sensing for cognitive radios. in Proceedings Asilomar Conference on Signals, Systems, and Computers, 772-776 (2004)

25. ECY Peh, Y-C Liang, YL Guan, Y Zeng, Optimization of cooperative sensing in cognitive radio networks: a sensing-throughput tradeoff view. IEEE Trans Veh Technol. 58(9), 5294-5299 (2009)

\section{doi:10.1186/1687-1499-2011-208}

Cite this article as: Yu et al:: Optimization of cooperative spectrum sensing with sensing user selection in cognitive radio networks. EURASIP Journal on Wireless Communications and Networking 2011 2011:208. 